\title{
New synthesized polyoxygenated diarylheptanoids suppress lipopolysaccharide-induced neuroinflammation
}

\author{
Anna Santarsiero a, Antonella Bochicchio a, Maria Funicello a, Paolo Lupattelli a, Sabine Choppin

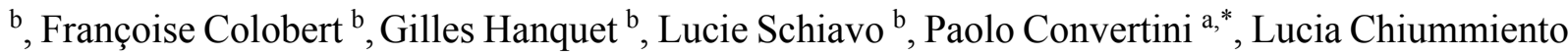 \\ a, ${ }^{*}$, and Vittoria Infantino ${ }^{\text {a }}$ \\ ${ }^{a}$ Department of Science, University of Basilicata, Via dell'Ateneo Lucano 10, 85100 Potenza, \\ Italy.
}

${ }^{\mathrm{b}}$ Université de Strasbourg, Université de Haute-Alsace, CNRS, UMR 7042-LIMA, ECPM, 25 Rue Becquerel, Strasbourg 67087, France

* Corresponding authors: Department of Science, Via dell'Ateneo Lucano 10, 85100 Potenza, Italy; paolo.convertini@uky.edu, $\quad+39 \quad 0971206102 \quad$ (Paolo Convertini); lucia.chiummiento@unibas.it, ,+390971205492 (Lucia Chiummiento).

\begin{abstract}
In neurodegenerative diseases, such as Alzheimer's disease, Huntington's disease, Parkinson's disease and multiple sclerosis, neuroinflammation induced by the microglial activation plays a crucial role. In effort to develop effective anti-neuroinflammatory compounds, different new linear polyoxygenated diarylheptanoids were synthesized. In LPS-triggered BV-2 microglial cells their ability to reduce the concentration of IL- 6 and TNF- $\alpha$ pro-inflammatory cytokines was evaluated. Moreover, their effect on NF-kB and ATP citrate lyase (ACLY), a recently emerged target of metabolic reprogramming in inflammation, was assessed. Finally, we turn our attention to
\end{abstract}


inflammatory mediators derived from the cleavage of citrate catalyzed by ACLY: prostaglandin $\mathrm{E}_{2}$, nitric oxide and reactive oxygen species. All compounds showed null or minimal cytotoxicity; most of them had a great anti-neuroinflammatory activity. Diarylheptanoids $\mathbf{6 b}$ and $\mathbf{6 c}$, bearing a halide atom and benzyl ether protective groups, exhibited the best effect since they blocked the secretion of all inflammatory mediators analyzed and reduced NF-kB and ATP citrate lyase protein levels.

Keywords: neuroinflammation; microglia; diarylheptanoids; anti-neuroinflammatory activity; $\mathrm{NF}-\kappa \mathrm{B}, \mathrm{ATP}$ citrate lyase

\section{Introduction}

Chronic neuroinflammation has a pivotal role in the onset and progression of a broad range of neurodegenerative diseases such as Alzheimer's disease, Huntington's disease, Parkinson's disease and multiple sclerosis [1]. Neuroinflammation is a consequence of the activation of microglia, the resident immune cells of the central nervous system. Microglial cells influence brain development and constantly survey the microenvironment of parenchyma, but they also act as the first line of defence against injury and invading pathogens. When the homeostasis is disrupted, microglia becomes activated and undergoes morphological and phenotypical changes into one of the two extremes of polarization: the classically activated M1 or alternatively activated M2 macrophages. M1 microglia secretes pro-inflammatory cytokines (e.g. TNF- $\alpha$, IL-1 $\beta$, IL-6), chemokines, prostaglandin $\mathrm{E}_{2}$ $\left(\mathrm{PGE}_{2}\right)$, reactive oxygen species (ROS), and reactive nitrogen species [2], which elicit 
detrimental effects on adjacent neurons and further exacerbate tissue inflammation and damage. By contrast, in M2 microglial cells the expression of anti-inflammatory mediators is up-regulated and they contribute in neuroprotection and tissue recovery and remodelling [3]. Therefore, neuroinflammation is a double-edged sword because of its beneficial or harmful effects on central nervous system, which depend on the type of microglia activation and the duration of inflammatory response. In fact, acute events scavenge harmful substances playing a protective role for nervous tissue while chronic ones are always dangerous due to the production and release of pro-inflammatory and cytotoxic factors [4]. In neurodegenerative diseases the equilibrium between microglial surveillance and activation is disrupted and growing evidence demonstrates that the control of microglia activation could attenuate their severity [5]. Therefore, the discovery of antineuroinflammatory compounds able to inhibit the microglia activation could be helpful in neurodegenerative disorders.

Diarylheptanoids (C6-C7-C6), a class of oxygenated compounds with a 1,7diphenylheptane skeleton, have been isolated from wood, bark or leaves of many hardwoods including Betulaceae, Leguminosae, Myricaceae and Proteaceae. They are increasingly recognized as potential therapeutic agents for their wide spectrum of biological and pharmacological activities such as anti-inflammatory, antioxidant, antitumor, estrogenic, leishmanicidal, melanogenesis, hepatoprotective, neuroprotective proprieties [6]. To this class of natural products belongs myricanol, a cyclic and not symmetric diarylheptanoid extracted from Myrica species, and curcumin, the main curcuminoid isolated from Curcuma longa (Fig. 1). It has been shown that myricanol reduces the levels of the microtubuleassociated protein tau resulting in anti-Alzheimer's disease effect [7] and curcumin exerts neuroprotective and anti-inflammatory activities [8-10]. In particular, curcumin inhibits 
$\mathrm{PGE}_{2}$, nitric oxide (NO), and tumor necrosis factor $\alpha$ (TNF- $\alpha$ ) release following lipopolysaccharide (LPS) stimulation in RAW 264.7 murine macrophages [11] and switches the polarization of LPS-activated BV2 microglial cells from M1 phenotype to a predominantly M2 phenotype [12].

Taking into consideration the biological importance of linear diarylheptanoids in general, and myricanol and curcumin in particular, we planned a straightforward access to a series of linear diarylheptanoid derivatives using the cross-metathesis reaction on readily available precursors $\mathbf{1 a}, \mathbf{b}, \mathbf{2 a}, \mathbf{b}$ and $\mathbf{3}$ [13-15]. Terminal olefins 1-3 [14] were easily prepared and used to give access to new linear diarylheptanoids and to the cyclic one, myricanol. Herein some of these were differently protected, halogenated and reduced. Eleven of the new synthesized diarylheptanoids were tested on a cell line model of neuroinflammation: LPS-triggered BV-2 cells. Their effect on different mediators of inflammation was evaluated in comparison with curcumin and celecoxib, a COX-2 selective non-steroidal anti-inflammatory drug (Fig. 1). Celecoxib, in a rat model with LPStriggered neuroinflammation, significantly decreased the rate of microglia activation and levels of IL-1 $\beta$ and TNF- $\alpha$ pro-inflammatory cytokines [16]. Finally, the ability of the new synthesized diarylheptanoids to reduce NF-kB and ATP citrate lyase (ACLY) - a metabolic pro-inflammatory enzyme - was also investigated.

\section{Material and Methods}

\subsection{Cell culture, treatment and cell viability assay}

The immortalized murine BV-2 microglial cell line was maintained in Roswell Park Memorial Institute 1640 Medium supplemented with 10\% heat inactivated fetal bovine 
serum, $4 \mathrm{mM}$ L-Glutamine, $100 \mathrm{U} / \mathrm{ml}$ penicillin and $100 \mu \mathrm{g} / \mathrm{ml}$ streptomycin at $37^{\circ} \mathrm{C}$ and $5 \% \mathrm{CO}_{2}$. Where indicated BV-2 cells were activated with $200 \mathrm{ng} / \mathrm{ml}$ LPS from Salmonella enterica serotype typhimurium (LPS, Sigma-Aldrich, St. Louis, MO, United States). Cell viability was evaluated by using CellTiter $96{ }^{\circledR}$ Non-Radioactive Cell Proliferation Assay (Promega, Madison, WI, USA) according to manufacturer's instructions. The details are given in Supplementary data.

\subsection{Enzyme-linked immunosorbent assay (ELISA)}

The concentration of pro-inflammatory cytokines IL-6 (Cat. $\mathrm{n}^{\circ}$ 32670069U1, ImmunoTools, Friesoythe, Germany) and TNF- $\alpha$ (Cat. n 32673019U1, ImmunoTools) in cell culture media was measured with ELISA kits following the manufacturer's recommendations. The levels of $\mathrm{PGE}_{2}$ were quantified by using Detect $X ®$ Prostaglandin E2 High Sensitivity Immunoassay Kit (Arbor Assays, Ann Arbor, MI, USA) as per manufacturer's protocol.

\subsection{ROS and NO detection}

Reactive oxygen species and nitric oxide levels were measured by using 6-Carboxy-2',7'Dichlorodihydrofluorescein Diacetate and 4-Amino-5-Methylamino-2', 7'Difluorofluorescein Diacetate (Thermo Fisher Scientific, San Jose, CA, USA), respectively, as previously described [17].

\subsection{Western blotting}


Western blotting experiments were carried-out as reported previously [18] using anti-NFkB/p65 (ab7970), anti-ATP citrate lyase (ab157098) or anti- $\beta$-actin (ab8227) primary antibodies (Abcam, Cambridge, MA). More details are reported in Supplementary data.

\subsection{Statistical analysis}

Statistical significance of differences was determined by using Student's t-test. Results are shown as means $\pm \mathrm{SD}$ of, at least, four independent experiments. Differences were considered as significant $(* \mathrm{p}<0.05)$, very significant $(* * \mathrm{p}<0.01)$ and highly significant $(* * * \mathrm{p}<0.001)$

\section{Results}

\subsection{Preparation of diarylheptanoids 4-6}

The requisite allylphenols $\mathbf{1 a}$ and $\mathbf{1 b}$ were accessed in two steps from commercially available 2,3dimethoxyphenol [13]. Coupling partners $\mathbf{2 a}, \mathbf{b}$ and $\mathbf{3}$ were readily prepared from commercial methyl 3-(4-benzyloxyphenyl)propanoate [14, 15]. With the key fragments $\mathbf{1 a}, \mathbf{1 b}, \mathbf{2 a}, \mathbf{2 b}$ and $\mathbf{3}$ in hands, we attempted cross metathesis (CM) reactions as depicted in Scheme $1[14,19,20]$. Hence, treatment of $\mathbf{1 a}$ ( 4 equiv.) with $\mathbf{2 a}$ or $\mathbf{2} \mathbf{b}$ (1 equiv.) in the presence of G-II catalyst $(5 \mathrm{~mol} \%)$ in dichloromethane from $-78^{\circ} \mathrm{C}$ to room temperature provided diarylheptanoid $\mathbf{4 a}$ and $\mathbf{4 c}$ in a gratifying $81 \%$ and $83 \%$ yield, respectively, if compared to the examples of $\mathrm{CM}$ at low temperature reported in literature [21].

Addition of G-II catalyst at $-78^{\circ} \mathrm{C}$ was mandatory to obtain good yields. Excess of partner 1a and its homocoupled were almost fully recovered after chromatography.

Cross metathesis between $\mathbf{1 b}$ and $\mathbf{2 a}$ afforded coupling product $\mathbf{4 b}$ in only $35 \%$ yield without formation of homocoupling product resulting from dimerization of $\mathbf{1 b}$. While positive effect 
of free $\mathrm{OH}$ (phenol or alcohol) on $\mathrm{CM}$ reactions, already reported in literature [22-24], could explain the excellent yield obtained to produce $\mathbf{4 a}$ (81\% yield), an opposite behavior was observed in the preparation of compound $\mathbf{4 b}$ (Scheme 1) despite the presence of two free $\mathrm{OH}$ on the starting catechol 1 1b.

Probably the lower solubility of catechol $\mathbf{1 b}$ in DCM at $-78^{\circ} \mathrm{C}$ prevented the metathesis reaction which started only increasing the temperature $\left(20^{\circ} \mathrm{C}\right)$. No dimerization of $\mathbf{1 b}$ was observed or after column chromatography. Moreover reaction between $\mathbf{1 a}$ and $\mathbf{2 b}$ gave rise to compound $\mathbf{4 c}$ in high yield (82\%) and cross metathesis between $\mathbf{1 a}$ and $\mathbf{3}$ afforded alkene $4 e$ in only $32 \%$ of yield.

Subsequent transformations were performed in order to have different protections and different functionalities(double bond, ketone or halide) on the same parent scaffold. Therefore, the acetylation of $\mathbf{4 a}$ by $\mathrm{Ac}_{2} \mathrm{O}$ in pyridine afforded $\mathbf{4 d}$ in a quantitative yield, while the methylation of $4 \mathbf{e}$ furnished the allylketone $4 \mathbf{f}$ in $30 \%$ of yield.

Hydrogenation of the double-bond and concurrent debenzylation of $4 \mathbf{a}$ resulted in the quantitative formation of $\mathbf{5 a}$ which was selectively protected on phenols as methyl ethers 5d. Chemoselective hydrogenation of $\mathbf{4 c}$ by $o-\mathrm{NO}_{2} \mathrm{PhSO}_{2} \mathrm{Cl}$ and $\mathrm{N}_{2} \mathrm{H}_{2}$ [25] furnished $\mathbf{5 b}$ in high yield, preserving the benzyl protecting group and the iodine atom of $\mathbf{4 c}$. Oxidation of compound 5d using DMP in DCM afforded the ketone 5e with 63\% yield. Compounds 5a and $\mathbf{5 b}$ were completely protected on phenol and carbinol functions as tribenzylethers $6 \mathbf{a}$ and $\mathbf{6 b}$ using $\mathrm{BnBr}, \mathrm{NaH}, \mathrm{NaI}$ in DMF. Subsequently regioselective bromination of $\mathbf{6 a}$ furnished the mono-brominated $\mathbf{6 c}$ and the di-brominated $\mathbf{6 d}$ in quantitative yield when 1.2 or 2.4 equiv of NBS was used, respectively.

\subsection{Novel polyoxygenated diarylheptanoids do not affect microglial cell viability}


BV-2 microglial cells were treated with curcumin, celecoxib or the novel polyoxygenated diarylheptanoids at $0.01,0.1$ and $1 \mu \mathrm{M}$ and their cytotoxic effects were determined by MTT assay after 72 hours (Fig. S1). Curcumin and celecoxib as well as the new-synthesized diarylheptanoids exhibited minimal or null toxic effect. We chose the lowest concentration $(0.01 \mu \mathrm{M})$ as optimal concentration to evaluate the anti-inflammatory activity.

\subsection{Impact of treatment with the linear polyoxygenated diarylheptanoids on IL-6, TNF- $\alpha$ and NF-KB}

When stimulated with lipopolysaccharide, the macrophages secrete different mediators among which IL-6 and TNF- $\alpha$ have a primary role for the initiation and propagation of inflammation [26]. Therefore, to probe the effect of the novel molecules on IL-6 and TNF- $\alpha$ secretion, BV-2 cells were stimulated with LPS in the presence or absence of each compound at the concentration of $0.01 \mu \mathrm{M}$ (Fig. 2). Twenty-four hours later, the levels of IL-6 and TNF- $\alpha$ were measured in cell culture media. LPS induced a significant release of IL-6 (Fig. 2A; LPS vs. CONTROL, $p<0.001$ ) and TNF- $\alpha$ (Fig. 2B; LPS vs. CONTROL, $\mathrm{p}<0.001$ ). Celecoxib resulted more active than curcumin in lowering IL6 levels compared to cells activated only with LPS (Fig. 2 A, $* * * p<0.001$ ). The majority of the tested compounds was able to reduce the secretion of IL-6; exceptions were molecules $4 \mathbf{a}, \mathbf{5 a}, \mathbf{5 c}$ and 5d, which had no significant effect (Fig. 2A). Interestingly, compound $\mathbf{4 b}$ was effective as celecoxib in lowering IL-6 levels, while compounds $\mathbf{5 e}$, $\mathbf{6 c}$ and $\mathbf{6 d}$ had an effect similar to curcumin. In the meantime, the diarylheptanoid $\mathbf{6 d}$ did not affect TNF- $\alpha$ release following LPS stimulation as well as compounds $\mathbf{4 b}, \mathbf{4 d}, \mathbf{5 a}, \mathbf{5 d}$ and $\mathbf{5 e}$ (Fig. 2B). The remaining molecules $\mathbf{4 a}$, 4f, 5c, $6 \mathbf{b}$ and $\mathbf{6 c}$ acted like celecoxib and curcumin in letting TNF- $\alpha$ concentration down (Fig. 2B, compounds vs. LPS, $* * * p<0.001)$. 
Then we investigated the effect of the new synthesized compounds on NF- $\mathrm{BB}$, a crucial transcription factor whose activation leads to the induction of multiple genes regulating the immune and the inflammatory responses [27]. Therefore, to verify if NF- $\kappa B$ could be a target for the polyoxygenated diarylheptanoids, LPS-triggered BV-2 cells were treated with the novel compounds, curcumin, or celecoxib. The protein levels of NF-kB subunit p65 were quantified via western blot. Surprisingly, all compounds were able to reduce the expression of NF- $\kappa B$ in the same manner as celecoxib and curcumin (Fig. 2C). Molecules $\mathbf{4 d}$ and $\mathbf{4 f}$ were the less active; however, they led to the reduction of about $30 \%$ in NF- $\kappa$ B protein levels with respect to BV-2 cells stimulated only with LPS (Fig. 2D).

\subsection{Effect of the novel polyoxygenated diarylheptanoids on ACLY expression and $\mathrm{PGE}_{2}$, NO and ROS production}

Since the new synthesized compounds reduced NF- $\kappa$ B expression and ACLY up-regulation in LPS-induced macrophages is under control of NF- $\mathrm{B}$ transcription factor [28], we evaluated the protein levels of ACLY (Fig. 3A). Of note, ACLY is a metabolic enzyme - recently identified as a new player of the immunometabolism - which splits glucose-derived citrate into acetyl-CoA and oxaloacetate (OAA). Acetyl-CoA provides units for lipid elongation, including arachidonic acid, precursor of prostaglandins. OAA leads to NADPH production, necessary for NADPH oxidase and iNOS to generate ROS and NO, respectively, in LPS-activated macrophages [29]. In BV-2 cells primed with LPS, curcumin and celecoxib were able to reduce ACLY expression levels (Fig. 3A). Among the new synthesized diarylheptanoids, the molecule $\mathbf{6 d}$ was the most active, even better than celecoxib, in lowering ACLY protein; on the contrary, molecules $\mathbf{4 b}, \mathbf{4 d}$ and $\mathbf{5 e}$ had no activity. The remaining compounds were able to reduce the expression of ACLY (Fig. 3A). 
Taking into consideration the inhibitory effect of ACLY by some of novel diarylheptanoids, we decided to monitor the levels of inflammatory mediators linked to the citrate pathway: $\mathrm{PGE}_{2}, \mathrm{NO}$ and ROS. When BV-2 microglial cells were activated with LPS we found a strong increase in PGE2 levels compared with untreated cells (Fig. 3B, CONTROL vs. LPS $p<0.001$ ). Celecoxib worked better than curcumin in modulating the production of $\mathrm{PGE}_{2}$, bringing the levels of $\mathrm{PGE}_{2}$ back to those of the untreated cells (Fig. 3B, CELECOXIB vs. LPS ***p<0.001). The majority of the tested diarylheptanoids acted as curcumin with the exception of compound $\mathbf{4 d}, \mathbf{4 f}$, $5 \mathbf{a}$ and $5 \mathrm{e}$. Molecules 4f and 5a had no effect: the levels of $\mathrm{PGE}_{2}$ in BV-2 cells were similar to cells treated only with LPS. On the contrary, compound $\mathbf{4 d}$ was the only tested diarylheptanoid more active than curcumin but less effective than celecoxib in reducing $\mathrm{PGE}_{2}$ levels (Fig. 3B).

Then, efforts were dedicated to NO and ROS evaluation. Intense production of both ROS and NO was observed in various diseases [30] and can be correlated to neurodegenerative disorders [31, 32]. Celecoxib resulted more active then curcumin in lowering NO and ROS levels compared to cells activated only with LPS (Fig. $3 \mathrm{C}-\mathrm{D}, \mathrm{p}<0.001$ ). The majority of the tested compounds had similar effect of curcumin with the exception of $\mathbf{6 b}$ and $\mathbf{6 c}$, the most active in the reduction of NO with an effect similar to celecoxib (Fig. 3C, 6b, 6c vs. LPS, $* * * p<0.001)$. By contrary, all the tested polyoxygenated diarylheptanoids, except 6d, showed a higher reduction of ROS levels with respect to the leading curcumin. Compounds $\mathbf{4 a}, \mathbf{4 d}, \mathbf{5 e}$ and $\mathbf{6 b}$ had the strongest inhibitor effect on LPSinduced ROS production in BV-2 cells (Fig. 3D, compounds vs. LPS, ${ }^{* *} \mathrm{p}<0.001$ ). Meanwhile, compound 5d was completely inactive: it even led to a significant increase in NO (Fig. 3C, $6 \mathbf{c}$ vs. LPS, $* * * p<0.001)$ as well as in ROS (Fig. 3D, 6c vs. LPS, $* * * p<0.001)$ levels.

\section{Discussion}


In our investigation, we reported the prospect of new eleven linear polyoxygenated diarylheptanoids as a source of alternative anti-neuroinflammatory compounds. To this end, they were synthesized according to known methodology [14] and screened in LPS-triggered BV-2 microglial cells. The tested diarylheptanoids could be classified into three class: a) with double bond (4a, 4b, 4d and 4f); b) without double bond (5a, 5c, 5d, and 5e) and c) with one or two halides on the aryl rings and completely protected on hydroxyl groups $(\mathbf{6 b}, \mathbf{6 c}$ and $\mathbf{6 d})$. Our findings showed that the acetylation of OHs had made more efficient compound $\mathbf{4 d}$ in lowering $\mathrm{PGE}_{2}, \mathrm{NO}$ and ROS levels with respect to $\mathbf{4 a}$ and $\mathbf{4 b}$ with one and two free phenolic group, respectively. Moreover, the presence of the carbonyl and the additional methyl ether in compound $\mathbf{4 f}$ made it with similar activity of $\mathbf{4 b}$ in reducing NO and ROS production and none inhibitory effect on $\mathrm{PGE}_{2}$ release. An opposite behaviour was observed in IL-6 and TNF- $\alpha$ secretion, where compound $\mathbf{4 d}$ was completely inactive while $\mathbf{4 f}$ had a high ability. Furthermore, molecule $4 \mathbf{b}$ reduced IL-6 concentration of about $30 \%$ similarly to celecoxib and 4a had a strong inhibitory effect on TNF- $\alpha$ secretion. From the second class of compounds $(\mathbf{5 a}, \mathbf{5 c}, \mathbf{5 d}, \mathbf{5 e})$ it is apparent that the absence of the double bond leads to a reduction in activity. For example, compound $\mathbf{5 d}$ did not have any inhibitory effect of IL-6 and TNF- $\alpha$ release and it even led an increase in NO and ROS levels with respect to BV-2 cells activated with LPS. Beneficial effects were found with benzyl ethers derivatives (third class) and in presence of just one halide (iodine or bromine) on one aryl ring, as in $\mathbf{6 b}$ and $\mathbf{6 c}$, respectively. These last compounds reduced the concentration of all inflammatory mediators analyzed and NF-kB and ACLY protein levels, while the presence of two bromine in the compound $\mathbf{6 d}$ induced loss of activity in lowering TNF- $\alpha$, NO and ROS levels. However, the compound $\mathbf{6 d}$ had the strongest inhibitory effect on ACLY. 
The great anti-neuroinflammatory activity of the compounds $\mathbf{6 b}$ and $\mathbf{6 c}$ could be linked to their lipophilic nature and to a possible passive membrane permeability. Despite molecular weights equal to 757 and $710 \mathrm{Da}$ and predicted partition coefficients, $\log \mathrm{P}$, of $11.88 \pm 0.47$ and $11.69 \pm 0.59$ for the molecules $\mathbf{6 b}$ and $\mathbf{6 c}$ respectively, anti-neuroinflammatory effects for compounds with similar values beyond Lipinski's Rule of 5 were recorded [33, 34]. In addition, it has been recently demonstrated that linear diarylheptanoids with a halide atom and the benzyl ethers as protective groups on the rings have biological activity [35]. Noteworthy, all polyoxygenated diarylheptanoids exhibited an inhibitory effect on NF- $\kappa \mathrm{B}$, transcription factor that regulates the expression of many genes involved in the inflammatory response among which IL-6, TNFo and ACLY genes [27, 28]. Therefore, the effects exerted by the novel diarylheptanoids on these latter targets could be whether direct inhibitory effects or consequence of NF-kB inhibition.

Surprisingly, the new synthesized molecules showed anti-neuroinflammatory features at $0.01 \mu \mathrm{M}$, a very low concentration respect to the reported cyclic ether diarylheptanoids [36] and respect to the natural linear diaryheptanoids [37] which showed anti-inflammatory and antioxidant proprieties at higher concentration, $30 \mu M$ and $13 \mu M$, respectively.

All these results shed light on chemical groups and/or modifications that look promising to improve anti-neuroinflammatory activity. Future investigation could demonstrate whether these compounds have other molecular targets in inflammation.

Founding This research was supported by Funding for Basic Research (FFABR) from the Italian Ministry of Education, University and Research and French government for LC PhD fellow. 


\section{References}

[1] E.E. Spangenberg, K.N. Green, Inflammation in Alzheimer's disease: Lessons learned from microglia-depletion models, Brain Behav Immun, 61 (2017) 1-11.

[2] D.W. Moss, T.E. Bates, Activation of murine microglial cell lines by lipopolysaccharide and interferon-gamma causes NO-mediated decreases in mitochondrial and cellular function, Eur $\mathrm{J}$ Neurosci, 13 (2001) 529-538.

[3] C.S. Subhramanyam, C. Wang, Q. Hu, S.T. Dheen, Microglia-mediated neuroinflammation in neurodegenerative diseases, Semin Cell Dev Biol, 94 (2019) 112-120.

[4] M.L. Block, Neuroinflammation: modulating mighty microglia, Nat Chem Biol, 10 (2014) 988989.

[5] V.H. Perry, C. Holmes, Microglial priming in neurodegenerative disease, Nat Rev Neurol, 10 (2014) 217-224.

[6] H. Lv, G. She, Naturally occurring diarylheptanoids, Nat Prod Commun, 5 (2010) 1687-1708.

[7] M.D. Martin, L. Calcul, C. Smith, U.K. Jinwal, S.N. Fontaine, A. Darling, K. Seeley, L. Wojtas, M. Narayan, J.E. Gestwicki, G.R. Smith, A.B. Reitz, B.J. Baker, C.A. Dickey, Synthesis, stereochemical analysis, and derivatization of myricanol provide new probes that promote autophagic tau clearance, ACS Chem Biol, 10 (2015) 1099-1109.

[8] M. Garcia-Alloza, L.A. Borrelli, A. Rozkalne, B.T. Hyman, B.J. Bacskai, Curcumin labels amyloid pathology in vivo, disrupts existing plaques, and partially restores distorted neurites in an Alzheimer mouse model, J Neurochem, 102 (2007) 1095-1104.

[9] W. Li, N.C. Suwanwela, S. Patumraj, Curcumin by down-regulating NF-kB and elevating Nrf2, reduces brain edema and neurological dysfunction after cerebral I/R, Microvasc Res, 106 (2016) 117-127. 
[10] E. Parada, I. Buendia, E. Navarro, C. Avendano, J. Egea, M.G. Lopez, Microglial HO-1 induction by curcumin provides antioxidant, antineuroinflammatory, and glioprotective effects, Mol Nutr Food Res, 59 (2015) 1690-1700.

[11] H.O. Pae, S.O. Jeong, H.S. Kim, S.H. Kim, Y.S. Song, S.K. Kim, K.Y. Chai, H.T. Chung, Dimethoxycurcumin, a synthetic curcumin analogue with higher metabolic stability, inhibits NO production, inducible NO synthase expression and NF-kappaB activation in RAW264.7 macrophages activated with LPS, Mol Nutr Food Res, 52 (2008) 1082-1091.

[12] J. Zhang, Y. Zheng, Y. Luo, Y. Du, X. Zhang, J. Fu, Curcumin inhibits LPS-induced neuroinflammation by promoting microglial M2 polarization via TREM2/ TLR4/ NF-kappaB pathways in BV2 cells, Mol Immunol, 116 (2019) 29-37.

[13] A. Bochicchio, R. Cefola, S. Choppin, F. Colobert, M.A. Di Noia, M. Funicello, G. Hanquet, I. Pisano, S. Todisco, L. Chiummiento, Selective Claisen rearrangement and iodination for the synthesis of polyoxygenated allyl phenol derivatives, Tetrahedron Letters, 57 (2016) 4053-4055.

[14] A. Bochicchio, L. Schiavo, L. Chiummiento, P. Lupattelli, M. Funicello, G. Hanquet, S. Choppin, F. Colobert, Convergent total synthesis of (+/-) myricanol, a cyclic natural diarylheptanoid, Org Biomol Chem, 16 (2018) 8859-8869.

[15] P. Massé, S. Choppin, L. Chiummiento, G. Hanquet, F. Colobert, Unintended Formation of a 26-Membered Cycle in the Course of a Novel Approach to Myricanol, a Strained [7,0]Metacyclophane, Synlett, 31 (2020) 559-564.

[16] L.W. Fan, A. Kaizaki, L.T. Tien, Y. Pang, S. Tanaka, S. Numazawa, A.J. Bhatt, Z. Cai, Celecoxib attenuates systemic lipopolysaccharide-induced brain inflammation and white matter injury in the neonatal rats, Neuroscience, 240 (2013) 27-38. 
[17] P. Convertini, A. Menga, G. Andria, I. Scala, A. Santarsiero, M.A. Castiglione Morelli, V. Iacobazzi, V. Infantino, The contribution of the citrate pathway to oxidative stress in Down syndrome, Immunology, 149 (2016) 423-431.

[18] V. Infantino, F. Dituri, P. Convertini, A. Santarsiero, F. Palmieri, S. Todisco, S. Mancarella, G. Giannelli, V. Iacobazzi, Epigenetic upregulation and functional role of the mitochondrial aspartate/glutamate carrier isoform 1 in hepatocellular carcinoma, Biochim Biophys Acta Mol Basis Dis, 1865 (2019) 38-47.

[19] S.J. Connon, S. Blechert, Recent developments in olefin cross-metathesis, Angew Chem Int Ed Engl, 42 (2003) 1900-1923.

[20] K.C. Nicolaou, P.G. Bulger, D. Sarlah, Metathesis reactions in total synthesis, Angew Chem Int Ed Engl, 44 (2005) 4490-4527.

[21] F. Rogano, G. Froidevaux, P. Rüedi, The Biomimetic Synthesis and First Characterization of the (+)- and (-)-Isocentrolobines, 2,6-cis- and 2,6-trans-Disubstituted Tetrahydro-2H-pyrans, Helvetica Chimica Acta, 93 (2010) 1299-1312.

[22] G.S. Forman, A.E. McConnell, R.P. Tooze, W. Janse van Rensburg, W.H. Meyer, M.M. Kirk, C.L. Dwyer, D.W. Serfontein, A Convenient System for Improving the Efficiency of FirstGeneration Ruthenium Olefin Metathesis Catalysts, Organometallics, 24 (2005) 4528-4542.

[23] Y.A. Lin, B.G. Davis, The allylic chalcogen effect in olefin metathesis, Beilstein J Org Chem, $6(2010)$ 1219-1228.

[24] T.K. Maishal, D.K. Sinha-Mahapatra, K. Paranjape, A. Sarkar, Effect of a proximal oxygen substituent on the efficacy of ruthenium-catalyzed cross-metathesis and RCM, Tetrahedron Letters, 43 (2002) 2263-2267. 
[25] B.J. Marsh, D.R. Carbery, One-Pot o-Nitrobenzenesulfonylhydrazide (NBSH) Formation-Diimide Alkene Reduction Protocol, The Journal of Organic Chemistry, 74 (2009) $3186-3188$.

[26] P. Singh, S. Kaur, A. Sharma, G. Kaur, R. Bhatti, TNF-alpha and IL-6 inhibitors: Conjugates of $\mathrm{N}$-substituted indole and aminophenylmorpholin-3-one as anti-inflammatory agents, Eur J Med Chem, 140 (2017) 92-103.

[27] T. Liu, L. Zhang, D. Joo, S.C. Sun, NF-kappaB signaling in inflammation, Signal Transduct Target Ther, 2 (2017).

[28] V. Infantino, V. Iacobazzi, F. Palmieri, A. Menga, ATP-citrate lyase is essential for macrophage inflammatory response, Biochem Biophys Res Commun, 440 (2013) 105-111.

[29] V. Infantino, C.L. Pierri, V. Iacobazzi, Metabolic Routes in Inflammation: The Citrate Pathway and its Potential as Therapeutic Target, Curr Med Chem, 26 (2019) 7104-7116.

[30] M. Valko, D. Leibfritz, J. Moncol, M.T. Cronin, M. Mazur, J. Telser, Free radicals and antioxidants in normal physiological functions and human disease, Int J Biochem Cell Biol, 39 (2007) 44-84.

[31] V. Calabrese, D. Boyd-Kimball, G. Scapagnini, D.A. Butterfield, Nitric oxide and cellular stress response in brain aging and neurodegenerative disorders: the role of vitagenes, In Vivo, 18 (2004) 245-267.

[32] C. Pimentel, L. Batista-Nascimento, C. Rodrigues-Pousada, R.A. Menezes, Oxidative stress in Alzheimer's and Parkinson's diseases: insights from the yeast Saccharomyces cerevisiae, Oxid Med Cell Longev, 2012 (2012) 132146.

[33] W.A. Banks, Characteristics of compounds that cross the blood-brain barrier, BMC Neurol, 9 Suppl 1 (2009) S3. 
[34] C.R. Pye, W.M. Hewitt, J. Schwochert, T.D. Haddad, C.E. Townsend, L. Etienne, Y. Lao, C. Limberakis, A. Furukawa, A.M. Mathiowetz, D.A. Price, S. Liras, R.S. Lokey, Nonclassical Size Dependence of Permeation Defines Bounds for Passive Adsorption of Large Drug Molecules, Journal of Medicinal Chemistry, 60 (2017) 1665-1672.

[35] A.F.M. Motiur Rahman, Y. Lu, H.J. Lee, H. Jo, W. Yin, M.S. Alam, H. Cha, A.A. Kadi, Y. Kwon, Y. Jahng, Linear diarylheptanoids as potential anticancer therapeutics: synthesis, biological evaluation, and structure-activity relationship studies, Arch Pharm Res, 41 (2018) 1131-1148.

[36] K. Maurent, C. Vanucci-Bacque, M. Baltas, A. Negre-Salvayre, N. Auge, F. Bedos-Belval, Synthesis and biological evaluation of diarylheptanoids as potential antioxidant and antiinflammatory agents, Eur J Med Chem, 144 (2018) 289-299.

[37] K. W. Woo, E. Moon, O. W. Kwon, S.O. Lee, S. Y. Kim, S. Z. Choi, M. W. Son, K.R. Lee, Antiinflammatory diarylheptanoids from rhizomes of Dioscorea nipponica, Biorg Med Chem Lett, 23 (2013) 3806-3809. 


\section{Figure legends}

Figure 1. Myricanol, curcumin, celecoxib and diarylheptanoids structures.

Figure 2. Effect of polyoxygenated diarylheptanoids on IL-6 and TNF- $\alpha$ release and NF-kB/ p65 expression levels. BV-2 cells were treated with DMSO (CONTROL) or $200 \mathrm{ng} / \mathrm{ml}$ LPS alone (LPS) or LPS plus $0.01 \mu \mathrm{M}$ celecoxib, curcumin, $4 \mathbf{a}, \mathbf{4 b}, \mathbf{4 d}, \mathbf{4 f}, 5 \mathbf{a}, \mathbf{5 c}, \mathbf{5 d}, 5 \mathbf{e}, \mathbf{6 b}, \mathbf{6 c}$ and $\mathbf{6 d}$ compounds. After 24 hours IL-6 (A) and TNF- $\alpha$ (B) concentrations were quantified. Means \pm S.D. of four duplicate independent experiments are shown; differences between CONTROL and LPS were highly significant $(\mathrm{p}<0.001$ Student's t-test $)$. Where indicated differences between LPS and cells treated with LPS plus compounds were significant $\left(* \mathrm{p}<0.05, * * \mathrm{p}<0.01,{ }^{* * *} \mathrm{p}<0.001\right.$, Student's t-test). C) The expression levels of subunit p65 of NF-kB were evaluated via western blotting. The intensity of immunolabelled protein bands depicted in $\mathbf{C}$ was quantified by densitometric scanning and NF-kB p65 levels were normalized against $\beta$-actin to have NF-kB p65 relative protein levels (D).

Figure 3. Effect of polyoxygenated diarylheptanoids on ACLY expression levels and PGE 2 , NO and ROS production. BV-2 microglial cells were treated with or $200 \mathrm{ng} / \mathrm{ml}$ LPS alone (LPS) or LPS plus $0.01 \mu \mathrm{M}$ celecoxib, curcumin, 4a, 4b, 4d, 4f, 5a, 5c, 5d, 5e, 6b, 6c and $\mathbf{6 d}$ compounds. A) By using specific antibodies, the expression levels of ACLY and $\beta$-actin were detected (upper panel). The intensity of immunolabelled protein bands was analyzed by densitometric scanning and ACLY levels were normalized against $\beta$-actin to have ACLY relative protein levels (lower panel). B-C-D) PGE2 concentration was measured after 48 hours of LPS treatment (B) while NO and ROS 24 hours later (C-D). Means \pm S.D. of four triplicate independent experiments are shown. In B-C-D differences between CONTROL and LPS were highly significant $(\mathrm{p}<0.001$, Student's $\mathrm{t}$ test). Where 
indicated, differences between LPS and cells treated with LPS plus compounds were significant $\left({ }^{*} p\right.$ $<0.05, * * \mathrm{p}<0.01, * * * \mathrm{p}<0.001$, Student's t-test).

Scheme 1 a) refs 13 and 14; b) Grubbs-II, DCM, $-78^{\circ} \mathrm{C}$ to rt, $81 \%$ yield $\mathbf{4 a}, 35 \%$ yield $\mathbf{4 b}, 82 \%$ yield 4c, 32\% yield 4e; c) $\mathrm{Ac}_{2} \mathrm{O}, \mathrm{Py}, \mathrm{rt}, 99 \%$; d) methylation of $\mathbf{4 e}$ and $5 \mathbf{a}$ by $\mathrm{MeI}, \mathrm{K}_{2} \mathrm{CO}_{3}$, acetone, 30\% yield $4 \mathbf{f}$ and $40 \%$ yield $\mathbf{5 d}$; e) hydrogenation of $\mathbf{4 a}$ by $\mathrm{H}_{2}, \mathrm{Pd} / \mathrm{C}, \mathrm{MeOH}, \mathrm{rt}, 1 \mathrm{~h}, 99 \%$ yield $\mathbf{5 a}$; f) hydrogenation of $\mathbf{4 c}$ by $0-\mathrm{NO}_{2} \mathrm{PhCl}, \mathrm{N}_{2} \mathrm{H}_{2} \cdot \mathrm{H}_{2} \mathrm{O}, \mathrm{ACN}, 0{ }^{\circ} \mathrm{C} 91 \%$ yield $\mathbf{5 c}$; g) benzylation of $\mathbf{5 a}$ by $\mathrm{BnBr}, \mathrm{K}_{2} \mathrm{CO}_{3}$, TBAI acetone, reflux , $99 \%$ yield $\mathbf{5 c}$; h) DMP, DCM, $63 \%$; i) benzylation of $5 \mathbf{a}$ and $\mathbf{5 b}$ by $\mathrm{BnBr}, \mathrm{NaH}, \mathrm{NaI}, \mathrm{DMF} 75 \%$ yield $\mathbf{6 a}$ and $71 \%$ yield $\mathbf{6 b} ; \mathrm{j})$ bromination of $\mathbf{6 a}$ by NBS ( 1.1 equiv or 2.2 equiv.), TFA, ACN 99\% yield $\mathbf{6 c}$ and 99\% yield $\mathbf{6 d}$. 